\title{
Statistical Mechanics of Nonlinear Nonequilibrium Financial Markets: Applications to Optimized Trading
}

\author{
Lester Ingber \\ Lester Ingber Research \\ P.O.B. 857, McLean, VA 22101, U.S.A. \\ ingber@alumni.caltech.edu
}

\begin{abstract}
A paradigm of statistical mechanics of financial markets (SMFM) using nonlinear nonequilibrium algorithms, first published in L. Ingber, Mathematical Modelling, 5, 343-361 (1984), is fit to multivariate financial markets using Adaptive Simulated Annealing (ASA), a global optimization algorithm, to perform maximum likelihood fits of Lagrangians defined by path integrals of multivariate conditional probabilities. Canonical momenta are thereby derived and used as technical indicators in a recursive ASA optimization process to tune trading rules. These trading rules are then used on out-ofsample data, to demonstrate that they can profit from the SMFM model, to illustrate that these markets are likely not efficient.
\end{abstract}

Keywords-Finance, Economics, Simulated Annealing, Statistical Mechanics 


\section{INTRODUCTION}

Over a decade ago, the author published a paper suggesting the use of newly developed methods of multivariate nonlinear nonequilibrium calculus to approach a statistical mechanics of financial markets (SMFM) [1]. That paper also gave a brief summary of the state of affairs of the equilibrium paradigm of financial markets. These methods were recently applied to interest-rate term-structure systems [2]. Still, for some time, the standard accepted paradigm of financial markets has been rooted in equilibrium processes [3]. There is a current effort by many to examine nonlinear and nonequilibrium processes in these markets, and this paper reinforces this point of view.

There are several issues that are clarified here, by presenting calculations of a specific trading model: (A) It is demonstrated how multivariate markets might be formulated in a nonequilibrium paradigm. (B) It is demonstrated that, despite possessing an algebra that was shunned a decade ago as being too complex, since then numerical methods of global optimization can be used to fit such SMFM models to data. (C) A variational principle possessed by SMFM permits derivation of technical indicators, such as canonical momenta, that can be used to describe deviations from most likely evolving states of the multivariate system. (D) These technical indicators can be embedded in realistic trading scenarios, to test whether they can profit from nonequilibrium in markets.

Section 2 outlines the formalism used to develop the nonlinear nonequilibrium SMFM model. Section 3 describes application of SMFM to SP500 cash and future data, using Adaptive Simulated Annealing (ASA) [4] to fit the short-time conditional probabilities developed in Section 2, and to establish trading rules by recursively optimizing with ASA, using optimized technical indicators developed from SMFM. These calculations were briefly mentioned in another ASA paper [5]. Section 4 is a brief conclusion.

\section{SMFM MODEL}

\subsection{Random Walk Model}

The following is a brief review of the rationale leading to SMFM close to its original presentation in 1984 [1]. Several studies imply that changing prices of many markets do not follow a random walk, that they may have long-term dependences in price correlations, and that they may not be efficient in 
quickly arbitraging new information [6-10].

(A) A random walk for returns, rate of change of prices over prices, is described by a Langevin equation with simple additive noise $\eta$, typically representing the continual random influx of information into the market.

$$
\begin{aligned}
& \dot{\Gamma}=-\gamma_{1}+\gamma_{2} \eta, \\
& \dot{\Gamma}=d \Gamma / d t, \\
& <\eta(t)>_{\eta}=0, \\
& <\eta(t), \eta\left(t^{\prime}\right)>_{\eta}=\delta\left(t-t^{\prime}\right),
\end{aligned}
$$

where $\gamma_{1}$ and $\gamma_{2}$ are constants, and $\Gamma$ is the logarithm of (scaled) price. From this equation, other models may be derived, such as the times-series model and the Kalman filter method of control theory [11], when care is taken to project physical states on observable space before eliminating irrelevant variables [12]. However, in the process of this transformation, the Markovian description typically is lost by projection onto a smaller state space [12,13]. In this context, price, although the most dramatic observable, may not be the only appropriate dependent variable or order parameter for the system of markets [14]. This possibility has also been called the "semistrong form of the efficient market hypothesis" [8].

This paper only considers nonlinear Gaussian noise, e.g., $\gamma_{2}$ not constant, also called "multiplicative" noise. These methods are not conveniently used for other sources of noise also considered by economists, e.g., Poisson processes [15] or Bernoulli processes [16,17]. For example, within limited ranges, log-normal distributions can approximate $1 / f$ distributions, and Pareto-Ĺevy tails may be modelled as subordinated log-normal distributions with amplification mechanisms [18].

(B) It is also necessary to explore the possibilities that a given market evolves in nonequilibrium, e.g., evolving irreversibly, as well as nonlinearly, e.g., $\gamma_{1,2}$ may be functions of $\Gamma$. Irreversibility, e.g., causality [19] and nonlinearity [20], have been suggested as processes necessary to take into account in order to understand markets, but modern methods of statistical mechanics now provide a more explicit paradigm to consistently include these processes in bona fide probability distributions. Reservations have been expressed about these earlier models at the time of their presentation [21]. 
(C) Besides assuming a rather specialized form for a Markovian process, Eq. (1) also assumes that real time is the proper independent variable. This is true for physical and most biophysical processes that have relatively continuous interactions [22-25], but for social and economic systems, some other density of relevant events might better describe the temporal evolution of the system.

For example, typically, $t$ is measured by a small time unit $\hat{t}$ that averages over a chosen number of ticks/trades and $\bar{t}$ is a macroscopic epoch. Reasonable values of $\hat{t}$ and $\bar{t}$ are on the order of minutes and days, respectively. A mesoscopic time scale $\tau, \bar{t}>\tau>\hat{t}$, and a "smoothness" parameter $\Gamma_{\gamma}$, a fraction of $\Gamma(\bar{t})$, are chosen to search and fit $\Gamma(t)$ to local minima and maxima. Thus a sequence of trades is taken to measure the independent temporal parameter of market $T$, and is mapped onto the variable $\Theta$, defined by integers $\rho: \Theta_{\rho}=\rho \tau+\Theta_{0}$.

Another reasonable scaling of $t$ onto a mesoscopic $\Theta^{\prime}$ would be to scale $t$ inversely to volume $V$ being traded, and to perform trades over a uniform mesh of $\Theta^{\prime}$. This would be one way of simulating an "average" trader.

(D) Developments in nonlinear nonequilibrium statistical mechanics in the late 1970's and their application to a variety of testable physical phenomena illustrate the importance of properly treating nonlinearities and nonequilibrium in systems where simpler analyses prototypical of linear equilibrium Brownian motion do not suffice [22-25].

\subsection{Statistical Mechanics of Large Systems}

Aggregation problems in nonlinear nonequilibrium systems typically are "solved" (accommodated) by having new entities/languages developed at these disparate scales in order to efficiently pass information back and forth [26,27]. This is quite different from the nature of quasi-equilibrium quasilinear systems, where thermodynamic or cybernetic approaches are possible. These approaches typically fail for nonequilibrium nonlinear systems.

In the late 1970's, mathematical physicists discovered that they could develop statistical mechanical theories from algebraic functional forms

$$
\begin{aligned}
& d r / d t=f_{r}(r, l)+\sum_{i} \hat{g}_{r}^{i}(r, l) \eta_{i}, \\
& d l / d t=f_{l}(r, l)+\sum_{i} \hat{g}_{l}^{i}(r, l) \eta_{i},
\end{aligned}
$$


where the $\hat{g}$ 's and $f$ 's are general nonlinear algebraic functions of the variables $r$ and $l$. These equations represent differential limits of discretized stochastic difference equations, e.g., Wiener noise $d W \rightarrow \eta d t$ [28]. The resulting stochastic differential equations (s.d.e.'s) are referred to as Langevin equations [28-33]. The $f$ 's are referred to as the (deterministic) drifts, and the square of the $\hat{g}$ 's are related to the diffusions (fluctuations or volatilities). In fact, the statistical mechanics can be developed for any number of variables, not just two. The $\eta$ 's are sources of Gaussian-Markovian noise, often referred to as "white noise." The inclusion of the $\hat{g}$ 's, called "multiplicative" noise, recently has been shown to very well mathematically and physically model other forms of noise, e.g., shot noise, colored noise, dichotomic noise [34-37]. Finite-jumps diffusions also can be included [38].

These new methods of nonlinear statistical mechanics only recently have been applied to complex large-scale physical problems, demonstrating that observed data can be described by the use of these algebraic functional forms. Success was gained for large-scale systems in neuroscience, in a series of papers on statistical mechanics of neocortical interactions [24,25,39-46], and in nuclear physics [47,48]. This methodology has been used for problems in combat analyses [27,49-52]. These methods have been suggested for financial markets [1], and applied to a term structure model of interest rates $[2,53]$.

Thus, now we can investigate various choices of $f$ 's and $\hat{g}$ 's to test algebraic functional forms. In science, this is a standard phenomenological approach to discovering and encoding knowledge and observed data, i.e., fitting algebraic functional forms which lend themselves to empirical interpretation. This gives more confidence when extrapolating to new scenarios, exactly the issue in building confidence in financial models.

The utility of these algebraic functional forms goes further beyond their being able to fit sets of data. There is an equivalent representation to the Langevin equations, called a "path-integral" representation for the long-time probability distribution of the variables. This short-time probability distribution is driven by a "Lagrangian," which can be thought of as a dynamic algebraic "cost" function. The path-integral representation for the long-time distribution possesses a variational principle, which means that simple graphs of the algebraic cost-function give a correct intuitive view of the most likely states of the variables, and of their statistical moments, e.g., heights being first moments (likely states) and widths being second moments (uncertainties). Like a ball bouncing about a terrain of hills and valleys, one can quickly visualize the nature of dynamically unfolding $r$ and $l$ states. 
Especially because we are trying to mathematically model sparse and poor data, different drift and diffusion algebraic functions can give approximately the same algebraic cost-function when fitting shorttime probability distributions to data. The calculation of long-time distributions permits a clear choice of the best algebraic functions, i.e., those which best follow the data through a predetermined long epoch of trading. Afterwards, if there are closely competitive algebraic functions, they can be more precisely assessed by calculating higher algebraic correlation functions from the probability distribution.

\subsection{Statistical Development}

When other order parameters in addition to price are included to study markets, Eq. (1) is accordingly generalized to a set of Langevin equations.

$$
\begin{aligned}
& \dot{M}^{G}=f^{G}+\hat{g}_{j}^{G} \eta^{j}, \\
& (G=1, \cdots, \Lambda), \\
& (j=1, \cdots, N), \\
& \dot{M}^{G}=d M^{G} / d \Theta, \\
& <\eta^{j}(\Theta)>_{\eta}=0, \\
& <\eta^{j}(\Theta), \eta^{j^{\prime}}\left(\Theta^{\prime}\right)>_{\eta}=\delta^{j j^{\prime}} \delta\left(\Theta-\Theta^{\prime}\right),
\end{aligned}
$$

where $f^{G}$ and $\hat{g}_{j}^{G}$ are generally nonlinear functions of mesoscopic order parameters $M^{G}, j$ is a microscopic index indicating the source of fluctuations, and $N \geq \Lambda$. The Einstein convention of summing over repeated indices is used. Vertical bars on an index, e.g., |j|, imply no sum is to be taken on repeated indices.

Via a somewhat lengthy, albeit instructive calculation, outlined in several other papers $[1,2,42]$, involving an intermediate derivation of a corresponding Fokker-Planck or Schr̈odinger-type equation for the conditional probability distribution $P\left[M(\Theta) \mid M\left(\Theta_{0}\right)\right]$, the Langevin rate Eq. (3) is developed into the more useful probability distribution for $M^{G}$ at long-time macroscopic time event $\Theta=(u+1) \theta+\Theta_{0}$, in terms of a Stratonovich path-integral over mesoscopic Gaussian conditional probabilities [29,54-57]. Here, macroscopic variables are defined as the long-time limit of the evolving mesoscopic system. 
The corresponding Schrodinger-type equation is [55,56]

$$
\begin{aligned}
& \partial P / \partial \Theta=\frac{1}{2}\left(g^{G G^{\prime}} P\right)_{, G G^{\prime}}-\left(g^{G} P\right)_{, G}+V \\
& g^{G G^{\prime}}=k_{T} \delta^{j k} \hat{g}_{j}^{G} \hat{g}_{k}^{G^{\prime}}, \\
& g^{G}=f^{G}+\frac{1}{2} \delta^{j k} \hat{g}_{j}^{G^{\prime}} \hat{g}_{k, G^{\prime}}^{G}, \\
& {[\cdots]_{, G}=\partial[\cdots] / \partial M^{G} .}
\end{aligned}
$$

This is properly referred to as a Fokker-Planck equation when $V \equiv 0$. Note that although the partial differential Eq. (4) contains equivalent information regarding $M^{G}$ as in the stochastic differential Eq. (3), all references to $j$ have been properly averaged over. I.e., $\hat{g}_{j}^{G}$ in Eq. (3) is an entity with parameters in both microscopic and mesoscopic spaces, but $M$ is a purely mesoscopic variable, and this is more clearly reflected in Eq. (4).

The path integral representation is given in terms of the Lagrangian $L$.

$$
\begin{aligned}
& P\left[M_{\Theta} \mid M_{\Theta_{0}}\right] d M(\Theta)=\int \cdots \int \underline{D} M \exp (-S) \delta\left[M\left(\Theta_{0}\right)=M_{0}\right] \delta\left[M(\Theta)=M_{\Theta}\right], \\
& S=k_{T}^{-1} \min _{\Theta_{0}}^{\Theta} d \Theta^{\prime} L \\
& \underline{D} M=\lim _{u \rightarrow \infty} \prod_{\rho=1}^{u+1} g^{1 / 2} \prod_{G}(2 \pi \theta)^{-1 / 2} d M_{\rho}^{G}, \\
& L\left(\dot{M}^{G}, M^{G}, \Theta\right)=\frac{1}{2}\left(\dot{M}^{G}-h^{G}\right) g_{G G^{\prime}}\left(\dot{M}^{G^{\prime}}-h^{G^{\prime}}\right)+\frac{1}{2} h_{; G}^{G}+R / 6-V, \\
& h^{G}=g^{G}-\frac{1}{2} g^{-1 / 2}\left(g^{1 / 2} g^{G G^{\prime}}\right)_{, G^{\prime}}, \\
& g_{G G^{\prime}}=\left(g^{G G^{\prime}}\right)^{-1}, \\
& g=\operatorname{det}\left(g_{G G^{\prime}}\right), \\
& h_{; G}^{G}=h_{, G}^{G}+\Gamma_{G F}^{F} h^{G}=g^{-1 / 2}\left(g^{1 / 2} h^{G}\right)_{, G},
\end{aligned}
$$




$$
\begin{aligned}
& \Gamma_{J K}^{F} \equiv g^{L F}[J K, L]=g^{L F}\left(g_{J L, K}+g_{K L, J}-g_{J K, L}\right), \\
& R=g^{J L} R_{J L}=g^{J L} g^{J K} R_{F J K L}, \\
& R_{F J K L}=\frac{1}{2}\left(g_{F K, J L}-g_{J K, F L}-g_{F L, J K}+g_{J L, F K}\right)+g_{M N}\left(\Gamma_{F K}^{M} \Gamma_{J L}^{N}-\Gamma_{F L}^{M} \Gamma_{J K}^{N}\right) .
\end{aligned}
$$

Mesoscopic variables have been defined as $M^{G}$ in the Langevin and Fokker-Planck representations, in terms of their development from the microscopic system labeled by $j$. The Riemannian curvature term $R$ arises from nonlinear $g_{G G^{\prime}}$, which is a bona fide metric of this parameter space [55]. Even if a stationary solution, i.e., $\dot{M}^{G}=0$, is ultimately sought, a necessarily prior stochastic treatment of $\dot{M}^{G}$ terms gives rise to these Riemannian "corrections." Even for a constant metric, the term $h_{; G}^{G}$ contributes to $L$ for a nonlinear mean $h^{G}$. V may include terms such as $\sum_{T^{\prime}} J_{T^{\prime} G} M^{G}$, where the Lagrange multipliers $J_{T^{\prime} G}$ are constraints on $M^{G}$, e.g., from other markets $T^{\prime}$, which are advantageously modelled as extrinsic sources in this representation; they too may be time-dependent. Using the variational principle below, $J_{T G}$ may also be used to constrain $M^{G}$ to regions where they are empirically bound. More complicated constraints may be affixed to $L$ using methods of optimal control theory [58]. With respect to a steady state $\bar{P}$, when it exists, the information gain in state $P$ is defined by

$$
\begin{aligned}
& \Upsilon[P]=\int \cdots \int \underline{D} M^{\prime} P \ln (P / \bar{P}), \\
& \underline{D} M^{\prime}=\underline{D} M / d M_{u+1} .
\end{aligned}
$$

In the economics literature, there appears to be sentiment to define Eq. (3) by the Ito, rather than the Stratonovich prescription. It should be noted that virtually all investigations of other physical systems, which are also continuous time models of discrete processes, conclude that the Stratonovich interpretation coincides with reality, when multiplicative noise with zero correlation time, modelled in terms of white noise $\eta^{j}$, is properly considered as the limit of real noise with finite correlation time [38]. The path integral succinctly demonstrates the difference between the two: The Ito prescription corresponds to the prepoint discretization of $L$, wherein $\theta \dot{M}(\Theta) \rightarrow M_{\rho+1}-M_{\rho}$ and $M(\Theta) \rightarrow M_{\rho}$. The Stratonovich prescription corresponds to the midpoint discretization of $L$, wherein $\theta \dot{M}(\Theta) \rightarrow M_{\rho+1}-M_{\rho}$ and $M(\Theta) \rightarrow \frac{1}{2}\left(M_{\rho+1}+M_{\rho}\right)$. In terms of the functions appearing in the Fokker-Planck Eq. (4), the Ito prescription of the prepoint discretized Lagrangian, $L_{I}$, is relatively simple, albeit deceptively so because 
of its nonstandard calculus.

$$
L_{I}\left(\dot{M}^{G}, M^{G}, \Theta\right)=\frac{1}{2}\left(\dot{M}^{G}-g^{G}\right) g_{G G^{\prime}}\left(\dot{M}^{G^{\prime}}-g^{G^{\prime}}\right)-V
$$

In the absence of a nonphenomenological microscopic theory, if the Ito prescription is proposed rather than the Stratonovich prescription, then this choice must be justified by numerical fits to data for each case considered.

There are several other advantages to Eq. (5) over Eq. (3). Extrema and most probable states of $M^{G}, \ll M^{G} \gg$, are simply derived by a variational principle, similar to conditions sought in previous studies [59]. In the Stratonovich prescription, necessary, albeit not sufficient, conditions are given by

$$
\begin{aligned}
& \delta_{G} L=L_{, G}-L_{, \dot{G}: \Theta}=0, \\
& L_{, \dot{G}: \Theta}=L_{, \dot{G} G^{\prime}} \dot{M}^{G^{\prime}}+L_{, \dot{G} \dot{G}^{\prime}} \ddot{M}^{G^{\prime}} .
\end{aligned}
$$

For stationary states, $\dot{M}^{G}=0$, and $\partial \bar{L} / \partial \bar{M}^{G}=0$ defines $\ll \bar{M}^{G} \gg$, where the bars identify stationary variables; in this case, the macroscopic variables are equal to their mesoscopic counterparts. [Note that $\bar{L}$ is not the stationary solution of the system, e.g., to Eq. (4) with $\partial P / \partial \Theta=0$. However, in some cases [40], $\bar{L}$ is a definite aid to finding such stationary states.] Typically, in other financial studies, only properties of stationary states are examined, but here a temporal dependence is included. E.g., the $\dot{M}^{G}$ terms in $L$ permit steady states and their fluctuations to be investigated in a nonequilibrium context. Note that Eq. (8) must be derived from the path integral, Eq. (5), which is at least one reason to justify its development.

In the language of nonlinear nonequilibrium thermodynamics [30], the thermodynamic forces are $\chi_{G}=S_{, G}$, where $S$ is the entropy, and the thermodynamic fluxes are $\dot{M}^{G}=g^{G G^{\prime}} \chi_{G^{\prime}}$. Although the fluxes are defined to be linearly related to the forces, $g^{G G^{\prime}}$ may be highly nonlinear in the state-variables $M^{G}$. The short-time Feynman Lagrangian $L$ can be expressed as the sum of the dissipation function $\phi\left(M^{G}, \dot{M}^{G}\right)=\frac{1}{2} g_{G G^{\prime}} \dot{M}^{G} \dot{M}^{G^{\prime}}$, the force function $\Psi\left(M^{G}, \chi_{G}\right)=\frac{1}{2} g^{G G^{\prime}} \chi_{G} \chi_{G^{\prime}}$, the potential term $-V$, and the (negative) rate of change of entropy $-\dot{S}\left(M^{G}\right)=-\chi_{G} \dot{M}^{G}$ : Then $L=\phi+\Psi-\dot{S}-V$ is the nonlinear nonequilibrium generalization of the Onsager-Machlup Lagrangian [60]. The variational equations insure that the equilibrium entropy is maximal, not necessarily a static equilibrium. Fluctuations over short time periods are introduced via variables $\eta_{G}=\partial L / \partial \dot{M}^{G}$ canonical to $M^{G}$, $\eta_{G}=g_{G G^{\prime}}\left(\dot{M}^{G^{\prime}}-g^{G^{\prime}}\right) \equiv g_{G G^{\prime}} \dot{M}^{G^{\prime}}-\chi_{G}$, interpreted as resulting from the nonequilibrium competition 
between the thermodynamic forces and fluxes. In the context of multiplicative Gaussian noise, the conditional probability of making the state-transition from $M_{\Theta}^{G}=M^{G}(\Theta)$ to $M_{\Theta+\theta}^{G}=M^{G}(\Theta+\theta)$ is then hypothesized to be $P\left[M_{\Theta+\theta}^{G} \mid M_{\Theta}^{G}\right] \propto \exp \left(-\frac{1}{2 k_{T}} \int_{\Theta}^{\Theta+\theta} d \Theta^{\prime} L\right) d \eta$. This machinery suffices to determine the macroscopic probability distribution [30]. For nonconstant $g_{G G^{\prime}}$ when $R \neq 0$, it should be noted that the Lagrangian corresponding to the most-probable path is not derived from the variational principle, but is directly related to $L$ [28].

To begin introducing economic theory, variables such as (logarithm) price can be postulated to be the basic state-variables. However, it is not clear how to precisely relate $L$ to classical economic equilibrium utility functions. It seems more reasonable to take economic microscopic models, usually formulated by differential equations of the state-variables, find regions of $M^{G}$ wherein multiplicative Gaussian noise modelling is appropriate, directly calculate $L$, and then to make the identification with thermodynamic forces, fluxes and entropy, if this is desired. It is argued here that, although the thermodynamic interpretation perhaps has aesthetic value, the prime utility of the statistical mechanical formulation of probability densities in terms of generalized Lagrangians is that detailed calculations can be performed of macroscopic evolutions of microscopic and mesoscopic mechanisms, even in highly nonlinear and nonequilibrium contexts.

\subsection{Algebraic Complexity Yields Simple Intuitive Results}

It must be emphasized that the output need not be confined to complex algebraic forms or tables of numbers. Because $L$ possesses a variational principle, sets of contour graphs, at different long-time epochs of the path-integral of $P$ over its variables at all intermediate times, give a visually intuitive and accurate decision-aid to view the dynamic evolution of the scenario. For example, this Lagrangian approach permits a quantitative assessment of concepts usually only loosely defined.

$$
\begin{aligned}
& \text { "Momentum" }=\Pi^{G}=\frac{\partial L}{\partial\left(\partial M^{G} / \partial \Theta\right)}, \\
& \text { "Mass" } g_{G G^{\prime}}=\frac{\partial^{2} L}{\partial\left(\partial M^{G} / \partial \Theta\right) \partial\left(\partial M^{G^{\prime}} / \partial \Theta\right)}, \\
& \text { "Force" }=\frac{\partial L}{\partial M^{G}},
\end{aligned}
$$




$$
\text { “F }=m a ": \delta L=0=\frac{\partial L}{\partial M^{G}}-\frac{\partial}{\partial \Theta} \frac{\partial L}{\partial\left(\partial M^{G} / \partial \Theta\right)},
$$

where $M^{G}$ are the variables and $L$ is the Lagrangian. These physical entities provide another form of intuitive, but quantitatively precise, presentation of these analyses. For example, daily newspapers use this terminology to discuss the movement of security prices. Here, we will use the canonical momenta as indicators to develop trading rules.

\subsection{Fitting Parameters}

The short-time path-integral Lagrangian of a $\Lambda$-dimensional system can be developed into a scalar "dynamic cost function," $C$, in terms of parameters, e.g., generically represented as $C(\tilde{\alpha})$,

$$
C(\tilde{\alpha})=L \Delta \Theta+\frac{\Lambda}{2} \ln (2 \pi \Delta \Theta)-\frac{1}{2} \ln g
$$

which can be used with the ASA algorithm, originally called Very Fast Simulated Reannealing (VFSR) [61] discussed below [4], to find the (statistically) best fit of parameters. The cost function for a given system is obtained by the product of $P$ 's over all data epochs, i.e., a sum of $C$ 's is obtained. Then, since we essentially are performing a maximum likelihood fit, the cost functions obtained from somewhat different theories or data can provide a relative statistical measure of their likelihood, e.g., $P_{12} \sim \exp \left(C_{2}-C_{1}\right)$.

If there are competing mathematical forms, then it is advantageous to utilize the path-integral to calculate the long-time evolution of $P[27,50]$. Experience has demonstrated that the long-time correlations derived from theory, measured against the observed data, is a viable and expedient way of rejecting models not in accord with observed evidence. Note that the use of the path integral is $a$ posteriori to and independent of the short-time fitting process, and is a subsidiary physical constraint on the mathematical models to judge their internal soundness and suitability for attempts to extrapolate to other trading scenarios.

\subsection{Numerical Methodology}

Two major computer codes have been developed to calculate these algebraic models, which are key tools for the use of this approach to estimate model parameters and price bonds. 
The first code, ASA [61], fits short-time probability distributions to observed data, using a maximum likelihood technique on the Lagrangian. This algorithm has been developed to fit observed data to a theoretical cost function over a $D$-dimensional parameter space [61], adapting for varying sensitivities of parameters during the fit. The annealing schedule for the "temperatures" (artificial fluctuation parameters) $T_{i}$ decrease exponentially in "time" (cycle-number of iterative process) $k$, i.e., $T_{i}=T_{i 0} \exp \left(-c_{i} k^{1 / D}\right)$.

Heuristic arguments have been developed to demonstrate that this algorithm is faster than the fast Cauchy annealing [62], $T_{i}=T_{0} / k$, and much faster than Boltzmann annealing [63], $T_{i}=T_{0} / \ln k$. To be more specific, the $k$ th estimate of parameter $\alpha^{i}$,

$$
\alpha_{k}^{i} \in\left[A_{i}, B_{i}\right]
$$

is used with the random variable $x^{i}$ to get the $k+1$ th estimate,

$$
\begin{aligned}
& \alpha_{k+1}^{i}=\alpha_{k}^{i}+x^{i}\left(B_{i}-A_{i}\right), \\
& x^{i} \in[-1,1] .
\end{aligned}
$$

The generating function is defined as

$$
\begin{aligned}
& g_{T}(x)=\prod_{i=1}^{D} \frac{1}{2 \ln \left(1+1 / T_{i}\right)\left(\left|x^{i}\right|+T_{i}\right)} \equiv \prod_{i=1}^{D} g_{T}^{i}\left(x^{i}\right), \\
& T_{i}=T_{i 0} \exp \left(-c_{i} k^{1 / D}\right) .
\end{aligned}
$$

Note that the use of $C$, the cost function given above, is not equivalent to doing a simple least squares fit on $M(\Theta+\Delta \Theta)$.

ASA has been applied to many problems by many people in many disciplines $[5,64,65]$. It is available via anonymous ftp from ftp.alumni.caltech.edu in directory/pub/ingber, which also can be accessed via the world-wide web (WWW) as http://www.alumni.caltech.edu/ ingber/.

The second code, PATHINT, develops the long-time probability distribution from the Lagrangian fit by the first code. A robust and accurate histogram-based (non-Monte Carlo) path-integral algorithm to calculate the long-time probability distribution has been developed to handle nonlinear Lagrangians $[44,50,66-70]$, 
The histogram procedure recognizes that the distribution can be numerically approximated to a high degree of accuracy as sum of rectangles at points $M_{i}$ of height $P_{i}$ and width $\Delta M_{i}$. For convenience, just consider a one-dimensional system. The above path-integral representation can be rewritten, for each of its intermediate integrals, as

$$
\begin{gathered}
P(M ; \Theta+\Delta \Theta)=\int d M^{\prime}\left[g_{s}^{1 / 2}(2 \pi \Delta \Theta)^{-1 / 2} \exp \left(-L_{s} \Delta \Theta\right)\right] P\left(M^{\prime} ; \Theta\right) \\
=\int d M^{\prime} G\left(M, M^{\prime} ; \Delta \Theta\right) P\left(M^{\prime} ; \Theta\right) \\
P(M ; \Theta)=\sum_{i=1}^{N} \pi\left(M-M_{i}\right) P_{i}(\Theta) \\
\pi\left(M-M_{i}\right)=\left\{\begin{array}{l}
0,\left(M_{i}-\frac{1}{2} \Delta M_{i-1}\right) \leq M \leq\left(M_{i}+\frac{1}{2} \Delta M_{i}\right), \\
1, \text { otherwise },
\end{array}\right.
\end{gathered}
$$

which yields

$$
\begin{aligned}
& P_{i}(\Theta+\Delta \Theta)=T_{i j}(\Delta \Theta) P_{j}(\Theta) \\
& T_{i j}(\Delta \Theta)=\frac{2}{\Delta M_{i-1}+\Delta M_{i}} \int_{M_{i}-\Delta M_{i-1} / 2}^{M_{i}+\Delta M_{i} / 2} d M \int_{M_{j}-\Delta M_{j-1} / 2}^{M_{j}+\Delta M_{j} / 2} d M^{\prime} G\left(M, M^{\prime} ; \Delta \Theta\right)
\end{aligned}
$$

$T_{i j}$ is a banded matrix representing the Gaussian nature of the short-time probability centered about the (varying) drift.

Fitting data with the short-time probability distribution, effectively using an integral over this epoch, permits the use of coarser meshes than the corresponding stochastic differential equation. The coarser resolution is appropriate, typically required, for numerical solution of the time-dependent pathintegral: By considering the contributions to the first and second moments of $\Delta M^{G}$ for small time slices $\theta$, conditions on the time and variable meshes can be derived [66]. The time slice essentially is determined by $\theta \leq \bar{L}^{-1}$, where $\bar{L}$ is the "static" Lagrangian with $d M^{G} / d \Theta=0$, throughout the ranges of $M^{G}$ giving the most important contributions to the probability distribution $P$. The variable mesh, a function of $M^{G}$, is optimally chosen such that $\Delta M^{G}$ is measured by the covariance $g^{G G^{\prime}}$, or $\Delta M^{G} \sim\left(g^{G G} \theta\right)^{1 / 2}$. 


\subsection{Application to Term-Structure Models}

An example of application of this formalism was to a term structure model developed for interest rates [2,53]. This model was developed with some sound economic theory, but the methodology presented here of using the methods of modern calculus, e.g., using the path-integral representation to define a maximum likelihood cost function has not yet been recognized by this community [71].

The pioneering Brennan-Schwartz (BS) model [72,73] was used to illustrate how to numerically implement this methodology $[2,53]$. Since "real time" is used in this model, the variable $t$ will be used instead of $\Theta$.

The BS model is summarized by:

$$
\begin{aligned}
& d r=\left[a_{1}+b_{1}(l-r)\right] d t+r \sigma_{1} d z_{1}, \\
& d l=\left[l\left(a_{2}+b_{2} r+c_{2} l\right)\right] d t+l \sigma_{2} d z_{2}, \\
& <d z_{i}>=0, i=\{1,2\}, \\
& <d z_{i}(t) d z_{j}\left(t^{\prime}\right)>=d t \delta\left(t-t^{\prime}\right), i=j, \\
& <d z_{i}(t) d z_{j}\left(t^{\prime}\right)>=\rho d t \delta\left(t-t^{\prime}\right), i \neq j, \\
& \delta\left(t-t^{\prime}\right)= \begin{cases}0, & t \neq t^{\prime}, \\
1, & t=t^{\prime},\end{cases}
\end{aligned}
$$

where $<$. $>$ denotes expectations.

These can be rewritten as Langevin equations (in the Itô prepoint discretization)

$$
\begin{aligned}
& d r / d t=a_{1}+b_{1}(l-r)+\sigma_{1} r\left(\gamma^{+} n_{1}+\operatorname{sgn} \rho \gamma^{-} n_{2}\right), \\
& d l / d t=l\left(a_{2}+b_{2} r+c_{2} l\right)+\sigma_{2} l\left(\operatorname{sgn} \rho \gamma^{-} n_{1}+\gamma^{+} n_{2}\right), \\
& \gamma^{ \pm}=\left[1 \pm\left(1-\rho^{2}\right)^{1 / 2}\right]^{1 / 2}, \\
& n_{i}=(d t)^{1 / 2} p_{i},
\end{aligned}
$$

where $p_{1}$ and $p_{2}$ are independent $[0,1]$ Gaussian distributions. 
The cost function $C$ is defined from the equivalent short-time probability distribution, $P$, for the above set of equations.

$$
\begin{aligned}
& P=g^{1 / 2}(2 \pi d t)^{-1 / 2} \exp (-L d t) \\
& =\exp (-C), \\
& C=L d t+\ln (2 \pi d t)-\frac{1}{2} \ln (g), \\
& L=\frac{1}{2} F^{\dagger} \underline{g} F, \\
& F=\left(\begin{array}{c}
d r / d t-\left(a_{1}+b_{1}(l-r)\right) \\
d l / d t-l\left(a_{2}+b_{2} r+c_{2} l\right)
\end{array}\right), \\
& \underline{g}=\frac{1}{k}\left(\begin{array}{lr}
\left(r \sigma_{1}\right)^{-2} & -\rho\left(r l \sigma_{1} \sigma_{2}\right)^{-1} \\
-\rho\left(r l \sigma_{1} \sigma_{2}\right)^{-1} & \left(l \sigma_{2}\right)^{-2}
\end{array}\right), \\
& g=\operatorname{det}(\underline{g}), \\
& k=1-\rho^{2} .
\end{aligned}
$$

$\underline{g}$, the metric in $\{r, l\}$-space, is the inverse of the covariance matrix,

$$
\underline{g}^{-1}=\left(\begin{array}{lr}
\left(r \sigma_{1}\right)^{2} & \rho r l \sigma_{1} \sigma_{2} \\
\rho r l \sigma_{1} \sigma_{2} & \left(l \sigma_{2}\right)^{2}
\end{array}\right) .
$$

As discussed above, the correct mesh for time, $d t$, in order that $P$ represent the Langevin equations (to order $\left.d t^{3 / 2}\right)$ is

$$
d t \leq 1 / \bar{L},
$$

where $\bar{L}$ is $L$ evaluated with $d s / d t=d l / d t=0$. If $d t$ is greater than $1 / \bar{L}$, then it is inappropriate to use $P$, and instead the path integral over intermediate states of folded short-time distributions must be calculated. In this context, it should be noted that the correct time mesh for the corresponding differential equations must be at least as small, since typically differentiation is a "sharpening" process. This will be noted in any discipline requiring numerical calculation, when comparing differential and integral representations of the same system. 
As reported [2,53], this methodology achieved a cost function a factor of 10 smaller than that calculated using the parameters given in the previous literature.

Since the author was given the data with the understanding that it would not be widely disseminated, they are not explicitly presented with this paper.

\section{FITTING SMFM TO SP500}

\subsection{Data Processing}

For the purposes of this paper, it suffices to consider a two-variable problem, SP500 prices of futures, $p^{1}$, and cash, $p^{2}$. Data included 251 points of 1989 and 252 points of 1990 daily closing data. (See Fig 1.) Time between data was taken as real time $t$, e.g., a weekend added two days to the time between data of a Monday and a previous Friday.

It was decided that relative data should be more important to the dynamics of the SMFM model than absolute data, and an arbitrary form was developed to preprocess data used in the fits,

$$
M^{i}(t)=p^{i}(t+\Delta t) / p^{i}(t)
$$

where $i=\{1,2\}=\{$ futures, $\operatorname{cash}\}$, and $\Delta t$ was the time between neighboring data points, and $t+\Delta t$ is the

current trading time. The ratio served to served to suppress strong drifts in the absolute data. (See Figs. 2 and 3.)

The Lagrangians fit to data below barely support the time scale of one day as being appropriate for for fitting and trading in this system. Certainly, weekly or monthly data would be too coarse.

\subsection{ASA Fits of SMFM to Data}

Two source of noise were assumed, so that the equations of this SMFM model are

$$
\frac{d M^{G}}{d t}=\sum_{G^{\prime}=1}^{2} f_{G^{\prime}}^{G} M^{G^{\prime}}+\sum_{i=1}^{2} \hat{g}_{i}^{G} \eta^{i}, G=\{1,2\} .
$$

The 8 parameters, $\left\{f_{G^{\prime}}^{G}, \hat{g}_{i}^{G}\right\}$ were all taken to be constants.

As discussed previously, the path-integral representation was used to define an effective cost function. Minimization of the cost function was performed using ASA. Some experimentation with the 
fitting process led to a scheme whereby after 1000 accepted states, the optimization was shunted over to a quasi-local code, the Broyden-Fletcher-Goldfarb-Shanno (BFGS) algorithm [74], to add another decimal of precision. If ASA was shunted over too quickly to BFGS, then poor fits were obtained, i.e., the fit stopped in a higher local minimum. Experience with ASA since 1993, and with its predecessor VFSR when these calculations were first performed in 1991, suggests that ASA might be as efficient as BFGS in the end stages of search for this particular problem, but this was not pursued here.

The values of the parameters obtained by this fitting process were: $f_{1}^{1}=0.135712, f_{1}^{2}=-0.135701$, $f_{2}^{1}=0.000190291, f_{2}^{2}=0.00047205, \hat{g}_{1}^{1}=-0.463577, \hat{g}_{1}^{2}=0.463687, \hat{g}_{2}^{1}=0.00156451, \hat{g}_{2}^{2}=$ 0.000767597 .

\subsection{ASA Fits of Trading Rules}

A simple model of trading was developed. The author is not a trader, and thus the model is obviously naive. (Yet, it worked well!) Two time-weighted moving averages, of long and short lengths $a_{L}$ and $a_{S}$ were defined. During each new epoch of $a_{L}$, always using the fits of the SMFM model described in the previous section as a zeroth order estimate, the parameters $\left\{f_{G^{\prime}}^{G}, \hat{g}_{i}^{G}\right\}$ were refit using data within each epoch. Averaged canonical momenta, i.e., using Eq. (9), were calculated for each new set of $a_{L}$ and $a_{S}$ epochs. Fluctuation parameters $\Delta q^{G}$ were defined, such that any change in trading position required that there was some reasonable information outside of these fluctuations that could be used as criteria for trading decisions. No trading was performed for the first few days of the year until the momenta could be calculated. Stop parameters also were placed to limit losses due to long or short positions in the futures market, $p \_l$ and $p \_s$, resp. Commissions of $\$ 70$ were paid every time a new trade of 100 units was taken. Thus, there were 6 trading parameters used in this example, $\left\{a_{L}, a_{S}, \Delta q^{G}, p_{-} l, p_{-} s\right\}$.

As listed in Table 1, the order of choices made for daily trading are as follows. A 0 represents no trading is performed until enough data is gathered, e.g., to calculate momenta. A 1 represents that all positions are closed out, e.g., at the end of the year. A 2 represents getting out of a long position. This was performed if the difference of the present price and the short-term averaged (within $a_{S}$ ) futures price, divided by the present price, was less than $p_{-} l$. A 3 represents getting out of a short position. This was performed if the negative of the difference of the present price and the short-term averaged futures price, 
divided by the present price, was less than $p_{-} l$. A 10 represents entering a long position, whether in a waiting or a short position; a current position was maintained. This was performed if the short-term averaged momenta of both cash and futures prices were both greater than 0 , and their long-term averaged momenta exceeded the $\Delta q^{G}$ fluctuation parameters. A -10 represents entering a short position, whether in a waiting or a long position; a current short position was maintained. This was performed if the shortterm averaged momenta of both cash and futures prices were both less than 0 , and their long-term averaged momenta were less than the $\Delta q^{G}$ fluctuation parameters. A 4 represents any remaining circumstance, getting out of any positions if they exist, and going into or maintaining a waiting position.

\subsubsection{In-sample ASA fits of trading rules}

For the data of 1989, recursive optimization was performed. The trading parameters were optimized in an outer shell, using the negative of the net yearly profit/loss as a cost function. This could have been weighted by something like the absolute value of maximum loss to help minimize risk, but this was not done here. The inner shell of optimization fine-tuning of the SMFM model was performed daily over the current $a_{L}$ epoch.

At first, ASA and shunting over to BFGS was used for each shell, but it was realized that good results could be obtained suing ASA and BFGS on the outer shell, and just BFGS on the inner shell (always using the ASA and BFGS derived zeroth order SMFM parameters as described above).

The trading parameters fit by this procedure were: $a_{L}=19, a_{S}=5, \Delta q^{1}=7.57644, \Delta q^{2}=53.549$, $p \_l=0.515714, p \_s=0.333581$.

\subsubsection{Out-of-sample SMFM trading}

The trading process described above was applied to the 1990 out-of-sample SP500 data. (See Fig. 1.) Note that 1990 was a "bear" market, while 1989 was a "bull" market. Thus, these two years had quite different overall contexts, and this was believed to provide a stronger test of this methodology than picking two years with similar contexts.

The inner shell of optimization was performed as described above for 1990 as well. Table 1 gives the daily results of trading. The summary of results is: cumulative profit $=\$ 24090$, number of profitable long positions $=8$, number of profitable short positions $=9$, number of losing long positions $=7$, number 
of losing short positions $=6$, maximum profit of any given trade $=\$ 8180$, maximum loss of any trade $=$ $-\$ 2145$, maximum accumulated profit during year $=\$ 26555$, maximum loss sustained during year $=$ $-\$ 5745$.

Fig. 4 gives graphs of momenta during 1989 and 1990. Comparing these to Figs. 2 and 3, note that there are more discernable cycles than are present in the ratio-variables. This suggests that the momenta more directly convey important information about the dynamics of the market than does the price.

Although only one variable, the futures SP500, was actually traded (the code can accommodate trading on multiple markets), note that the multivariable coupling to the cash market entered in three important ways: (1) The SMFM fits were to the coupled system, requiring a global optimization of all parameters in both markets to define the time evolution of the futures market. (2) The canonical momenta for the futures market is in terms of the partial derivative of the full Lagrangian; the dependency on the cash market enters both as a function of the relative value of the off-diagonal to diagonal terms in the metric, as well as a contribution to the drifts and diffusions from this market. (3) The canonical momenta of both markets were used as technical indicators for trading the futures market.

\subsubsection{Reversing data sets}

The same procedures described above were repeated, but using the 1990 SP500 data set for training and the 1989 data set for testing.

The values of the parameters obtained by this fitting process were: $f_{1}^{1}=0.138135, f_{1}^{2}=-0.138146$,

$f_{2}^{1}=1.37507 \mathrm{e}-05, f_{2}^{2}=0.000576185, \hat{g}_{1}^{1}=-0.460157, \hat{g}_{1}^{2}=0.460342, \hat{g}_{2}^{1}=0.000876352, \hat{g}_{2}^{2}=$ 0.00168462 .

The trading parameters fit by this procedure were: $a_{L}=22, a_{S}=4, \Delta q^{1}=20.3058, \Delta q^{2}=96.1126$, $p \_l=0.15634, p \_s=0.15898$.

The summary of results is: cumulative profit $=\$ 10265$, number of profitable long positions $=14$, number of profitable short positions $=5$, number of losing long positions $=11$, number of losing short positions $=11$, maximum profit of any given trade $=\$ 9605$, maximum loss of any trade $=-\$ 2170$, maximum accumulated profit during year $=\$ 14130$, maximum loss sustained during year $=-\$ 1270$. 


\section{CONCLUSION}

A complete sample scenario has been presented: (a) developing a multivariate nonlinear nonequilibrium model of financial markets; (b) fitting the model to data using methods of ASA global optimization; (c) deriving technical indicators to express dynamics about most likely states; (d) optimizing trading rules using these technical indicators; (e) trading on out-of-sample data to determine if steps (a)-(d) are at least sufficient to profit by the knowledge gained of these financial markets, i.e., these markets are not efficient.

Just based the models and calculations presented here, no comparisons can yet be made of any relative superiority of these techniques over other models of markets and other sets of trading rules. Rather, this exercise should be viewed as an explicit demonstration (1) that financial markets can be modeled as nonlinear nonequilibrium systems, and (2) that financial markets are not efficient and that they can be properly fit and profitably traded on real data. 


\section{REFERENCES}

1. L. Ingber, Statistical mechanics of nonlinear nonequilibrium financial markets, Math. Modelling 5 (6), 343-361 (1984).

2. L. Ingber, M.F. Wehner, G.M. Jabbour, and T.M. Barnhill, Application of statistical mechanics methodology to term-structure bond-pricing models, Mathl. Comput. Modelling 15 (11), 77-98 (1991).

3. R.C. Merton, Continuous-Time Finance, Blackwell, Cambridge, MA, (1992).

4. L. Ingber, Adaptive Simulated Annealing (ASA), Global optimization C-code, Lester Ingber Research, Chicago, IL, (1993).

5. L. Ingber, Simulated annealing: Practice versus theory, Mathl. Comput. Modelling 18 (11), 29-57 (1993).

6. T. F. Cargill and G. C. Rausser, Temporal price behavior in commodity futures markets, J. Finance 30, 1043-1053 (1975).

7. M. T. Greene and B. D. Fielitz, Long-term dependence in common stock returns, J. Fin. Econ. 4, 339-349 (1977).

8. M. C. Jensen, Some anomalous evidence regarding market efficiency, an editorial introduction, $J$. Finan. Econ. 6, 95-101 (1978).

9. B. B. Mandelbrot, When can price be arbitraged efficiently? A limit to the validity of the random walk and martingale models, Rev. Econ. Statist. 53, 225-236 (1971).

10. S. J. Taylor, Tests of the random walk hypothesis against a price-trend hypothesis, J. Finan. Quant. Anal. 17, 37-61 (1982).

11. E. Burmeister and K. D. Wall, Kalman filtering estimation of unobserved rational expectation with an application to the German hyperinflation, J. Econ. 20, 255-284 (1982).

12. K. Kishida, Physical Langevin model and the time-series model in systems far from equilibrium, Phys. Rev. A 25, 496-507 (1982).

13. K. Kishida, Equivalent random force and time-series model in systems far from equilibrium, $J$. Math. Phys. 25, 1308-1313 (1984). 
14. P. Brown, A. W. Kleidon, and T. A. Marsh, New evidence on the nature of size-related anomalies in stock prices, J. Fin. Econ. 12, 33-56 (1983).

15. R. C. Merton, Option pricing when underlying stock returns are discontinuous, J. Fin. Econ. 3, 125-144 (1976).

16. C. A. Ball and W. N. Torous, A simplified jump process for common stock returns, J. Fin. Quant. Analysis 18, 53-65 (1983).

17. J. C. Cox, S. A. Ross, and M. Rubenstein, Option pricing: A simplified approach, J. Fin. Econ. 7, 229-263 (1979).

18. E. W. Montroll and M. F. Shlesinger, Maximum entropy formalism, fractals, scaling phenomena, and $1 / f$ noise: A tale of tails, J. Stat. Phys. 32, 209-230 (1983).

19. C. W. J. Granger, Investigating causal relations by econometric models and cross-spectral methods, Econometrica 37, 424-438 (1969).

20. P. K. Clark, A subordinated stochastic process model with finite variance for speculative prices, Econometrica 41, 135-155 (1973).

21. B. B. Mandelbrot, Comments on: 'A subordinated stochastic process model with finite variance for speculative prices,' by Peter K. Clark, Econometrica 41, 157-159 (1973).

22. H. Haken, Synergetics, Springer, New York, (1983).

23. L. Ingber, Statistical mechanics of neocortical interactions. I. Basic formulation, Physica D 5, 83-107 (1982).

24. L. Ingber, Statistical mechanics of neocortical interactions. Dynamics of synaptic modification, Phys. Rev. A 28, 395-416 (1983).

25. L. Ingber, Statistical mechanics of neocortical interactions. Derivation of short-term-memory capacity, Phys. Rev. A 29, 3346-3358 (1984).

26. L. Ingber, Mesoscales in neocortex and in command, control and communications $\left(\mathrm{C}^{3}\right)$ systems, in Systems with Learning and Memory Abilities: Proceedings, University of Paris 15-19 June 1987, (Edited by J. Delacour and J.C.S. Levy), pp. 387-409, Elsevier, Amsterdam, (1988).

27. L. Ingber, Mathematical comparison of JANUS(T) simulation to National Training Center, in The Science of Command and Control: Part II, Coping With Complexity, (Edited by S.E. Johnson and 
A.H. Levis), pp. 165-176, AFCEA International, Washington, DC, (1989).

28. F. Langouche, D. Roekaerts, and E. Tirapegui, Functional Integration and Semiclassical Expansions, Reidel, Dordrecht, The Netherlands, (1982).

29. H. Dekker, Functional integration and the Onsager-Machlup Lagrangian for continuous Markov processes in Riemannian geometries, Phys. Rev. A 19, 2102-2111 (1979).

30. H. Grabert and M.S. Green, Fluctuations and nonlinear irreversible processes, Phys. Rev. A 19, 1747-1756 (1979).

31. R. Graham, Covariant formulation of non-equilibrium statistical thermodynamics, Z. Physik B26, 397-405 (1977).

32. R. Graham, Lagrangian for diffusion in curved phase space, Phys. Rev. Lett. 38, 51-53 (1977).

33. L.S. Schulman, Techniques and Applications of Path Integration, J. Wiley \& Sons, New York, (1981).

34. L. Ingber, Nonlinear nonequilibrium statistical mechanics approach to $\mathrm{C}^{3}$ systems, in 9 th MIT/ONR Workshop on $C^{3}$ Systems: Naval Postgraduate School, Monterey, CA, 2-5 June 1986, pp. 237-244, MIT, Cambridge, MA, (1986).

35. R.F. Fox, Uniform convergence to an effective Fokker-Planck equation for weakly colored noise, Phys. Rev. A 34, 4525-4527 (1986).

36. C. van der Broeck, On the relation between white shot noise, Gaussian white noise, and the dichotomic Markov process, J. Stat. Phys. 31, 467-483 (1983).

37. P. Colet, H.S. Wio, and M. San Miguel, Colored noise: A perspective from a path-integral formalism, Phys. Rev. A 39, 6094-6097 (1989).

38. C.W. Gardiner, Handbook of Stochastic Methods for Physics, Chemistry and the Natural Sciences, Springer-Verlag, Berlin, Germany, (1983).

39. L. Ingber, Statistical mechanics of neocortical interactions. EEG dispersion relations, IEEE Trans. Biomed. Eng. 32, 91-94 (1985).

40. L. Ingber, Statistical mechanics of neocortical interactions: Stability and duration of the 7+-2 rule of short-term-memory capacity, Phys. Rev. A 31, 1183-1186 (1985). 
41. L. Ingber and P.L. Nunez, Multiple scales of statistical physics of neocortex: Application to electroencephalography, Mathl. Comput. Modelling 13 (7), 83-95 (1990).

42. L. Ingber, Statistical mechanics of neocortical interactions: A scaling paradigm applied to electroencephalography, Phys. Rev. A 44 (6), 4017-4060 (1991).

43. L. Ingber, Generic mesoscopic neural networks based on statistical mechanics of neocortical interactions, Phys. Rev. A 45 (4), R2183-R2186 (1992).

44. L. Ingber, Statistical mechanics of neocortical interactions: Path-integral evolution of short-term memory, Phys. Rev. E 49 (5B), 4652-4664 (1994).

45. L. Ingber, Statistical mechanics of multiple scales of neocortical interactions, in Neocortical Dynamics and Human EEG Rhythms, (Edited by P.L. Nunez), pp. 628-681, Oxford University Press, New York, NY, (1995).

46. L. Ingber, Statistical mechanics of neocortical interactions: Multiple scales of EEG, in Frontier Science in EEG: Continuous Waveform Analysis (Electroencephal. clin. Neurophysiol. Suppl. 45), (Edited by R.M. Dasheiff and D.J. Vincent), pp. 79-112, Elsevier, Amsterdam, (1996).

47. L. Ingber, Path-integral Riemannian contributions to nuclear Schrödinger equation, Phys. Rev. D 29, 1171-1174 (1984).

48. L. Ingber, Riemannian contributions to short-ranged velocity-dependent nucleon-nucleon interactions, Phys. Rev. D 33, 3781-3784 (1986).

49. L. Ingber, Mathematical comparison of computer models to exercise data, in $1989 J D L C^{2}$ Symposium: National Defense University, Washington, DC, 27-29 June 1989, pp. 169-192, SAIC, McLean, VA, (1989).

50. L. Ingber, H. Fujio, and M.F. Wehner, Mathematical comparison of combat computer models to exercise data, Mathl. Comput. Modelling 15 (1), 65-90 (1991).

51. L. Ingber and D.D. Sworder, Statistical mechanics of combat with human factors, Mathl. Comput. Modelling 15 (11), 99-127 (1991).

52. L. Ingber, Statistical mechanics of combat and extensions, in Toward a Science of Command, Control, and Communications, (Edited by C. Jones), pp. 117-149, American Institute of Aeronautics and Astronautics, Washington, D.C., (1993). 
53. L. Ingber, Statistical mechanical aids to calculating term structure models, Phys. Rev. A 42 (12), 7057-7064 (1990).

54. K.S. Cheng, Quantization of a general dynamical system by Feynman's path integration formulation, J. Math. Phys. 13, 1723-1726 (1972).

55. R. Graham, Path-integral methods on nonequilibrium thermodynamics and statistics, in Stochastic Processes in Nonequilibrium Systems, (Edited by L. Garrido, P. Seglar, and P.J. Shepherd), pp. 82-138, Springer, New York, NY, (1978).

56. F. Langouche, D. Roekaerts, and E. Tirapegui, Discretization problems of functional integrals in phase space, Phys. Rev. D 20, 419-432 (1979).

57. F. Langouche, D. Roekaerts, and E. Tirapegui, Short derivation of Feynman Lagrangian for general diffusion process, J. Phys. A 113, 449-452 (1980).

58. P. Hagedorn, Non-Linear Oscillations, Oxford Univ., New York, NY, (1981).

59. R. C. Merton, An intertemporal capital asset pricing model, Econometrica 41, 867-887 (1973).

60. L. Onsager and S. Machlup, Fluctuations and irreversible processes, Phys. Rev. 91, 1505-1512 (1953).

61. L. Ingber, Very fast simulated re-annealing, Mathl. Comput. Modelling 12 (8), 967-973 (1989).

62. H. Szu and R. Hartley, Fast simulated annealing, Phys. Lett. A 122 (3-4), 157-162 (1987).

63. S. Kirkpatrick, C.D. Gelatt, Jr., and M.P. Vecchi, Optimization by simulated annealing, Science 220 (4598), 671-680 (1983).

64. M. Wofsey, Technology: Shortcut tests validity of complicated formulas, The Wall Street Journal 222 (60), B1 (1993).

65. L. Ingber, Adaptive simulated annealing (ASA): Lessons learned, Control and Cybernetics 25 (1), 33-54 (1996).

66. M.F. Wehner and W.G. Wolfer, Numerical evaluation of path-integral solutions to Fokker-Planck equations. I., Phys. Rev. A 27, 2663-2670 (1983).

67. M.F. Wehner and W.G. Wolfer, Numerical evaluation of path-integral solutions to Fokker-Planck equations. II. Restricted stochastic processes, Phys. Rev. A 28, 3003-3011 (1983). 
68. M.F. Wehner and W.G. Wolfer, Numerical evaluation of path integral solutions to Fokker-Planck equations. III. Time and functionally dependent coefficients, Phys. Rev. A 35, 1795-1801 (1987).

69. L. Ingber, Path-integral evolution of multivariate systems with moderate noise, Phys. Rev. E 51 (2), 1616-1619 (1995).

70. L. Ingber and P.L. Nunez, Statistical mechanics of neocortical interactions: High resolution pathintegral calculation of short-term memory, Phys. Rev. E 51 (5), 5074-5083 (1995).

71. B. Dietrich-Campbell and E. Schwartz, Valuing debt options, J. Finan. Econ. 16, 321-343 (1986).

72. M.J. Brennan and E.S. Schwartz, A continuous time approach to the pricing of bonds, J. Banking Finan. 3, 133-155 (1979).

73. M.J. Brennan and E.S. Schwartz, An equilibrium model of bond pricing and a test of market efficiency, J. Finan. Quant. Anal. 17, 301-329 (1982).

74. D.F. Shanno and K.H. Phua, Minimization of unconstrained multivariate functions, ACM Trans. Mathl. Software 2, 87-94 (1976). 


\section{FIGURE CAPTIONS}

Figure 1. SP500 cash and futures prices used used in the SMFM study.

Figure 2. SP500 ratios of prices for 1989 SP500 cash and futures.

Figure 3. SP500 ratios of prices for 1990 SP500 cash and futures.

Figure 4. SP500 momenta of futures prices for 1989 and 1990 SP500. The 1989 momenta were calculated after using 1990 data to fit the SMFM model. The 1990 momenta were calculated after using 1989 data to fit the SMFM model. 


\section{TABLE CAPTIONS}

Table 1. Results of SMFM Trading on 1990 SP500. The first column gives the date of the trade. The second column gives the action taken: A 0 represents no trading is performed until enough data is gathered, e.g., to calculate momenta. A 1 represents that all positions are closed out, e.g., at the end of the year. A 2 represents getting out of a long position. A 3 represents getting out of a short position. A 10 represents entering a long position, whether in a waiting or a short position. A -10 represents entering a short position, whether in a waiting or a long position. A 4 represents any remaining circumstance, getting out of any positions if they exist, and going into or maintaining a waiting position. The third column gives the current futures price. The fourth column gives the derived canonical momenta of the futures price. The fifth column gives the day-to-day change in profit or loss. The sixth column gives the accumulated profit or loss. 


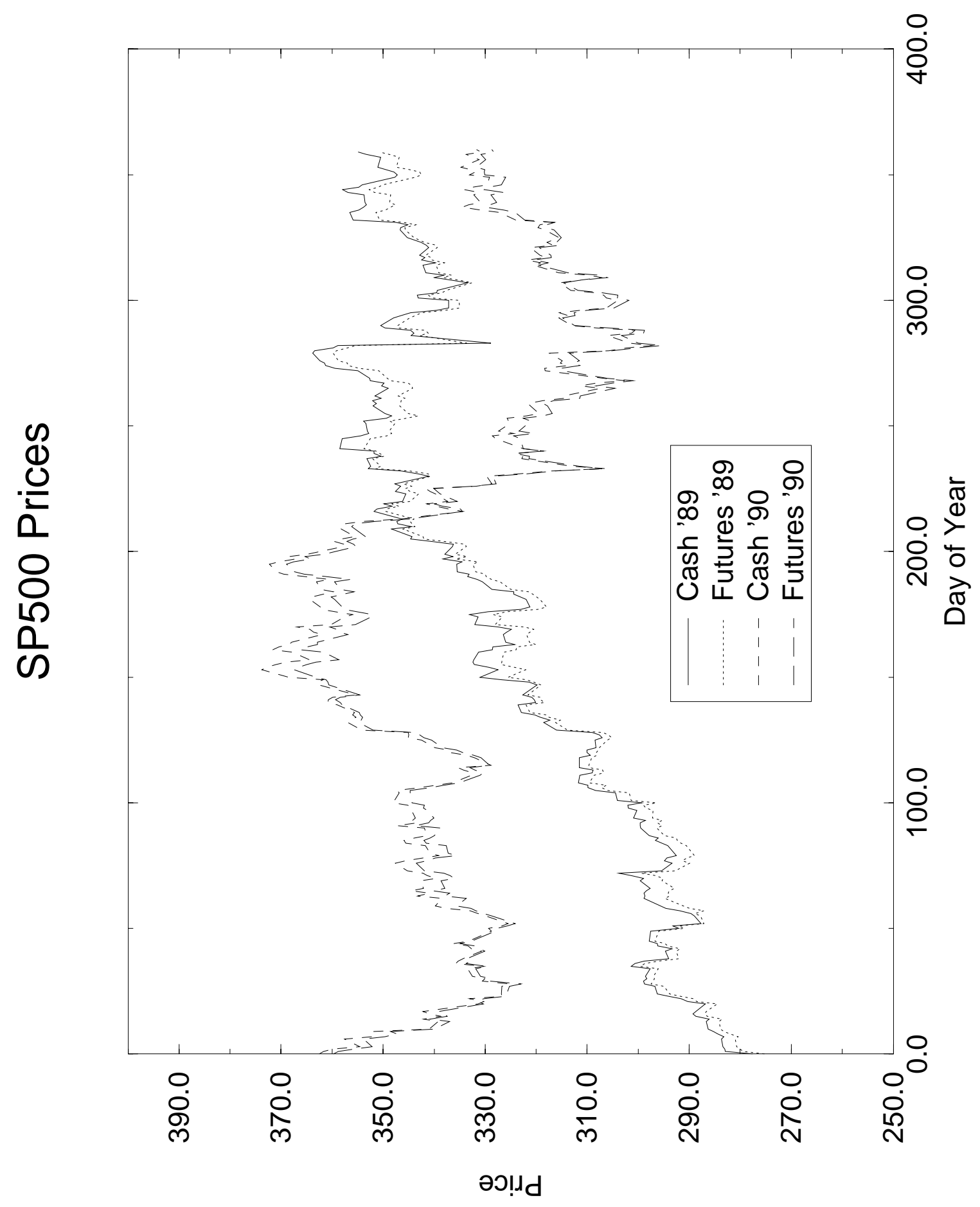




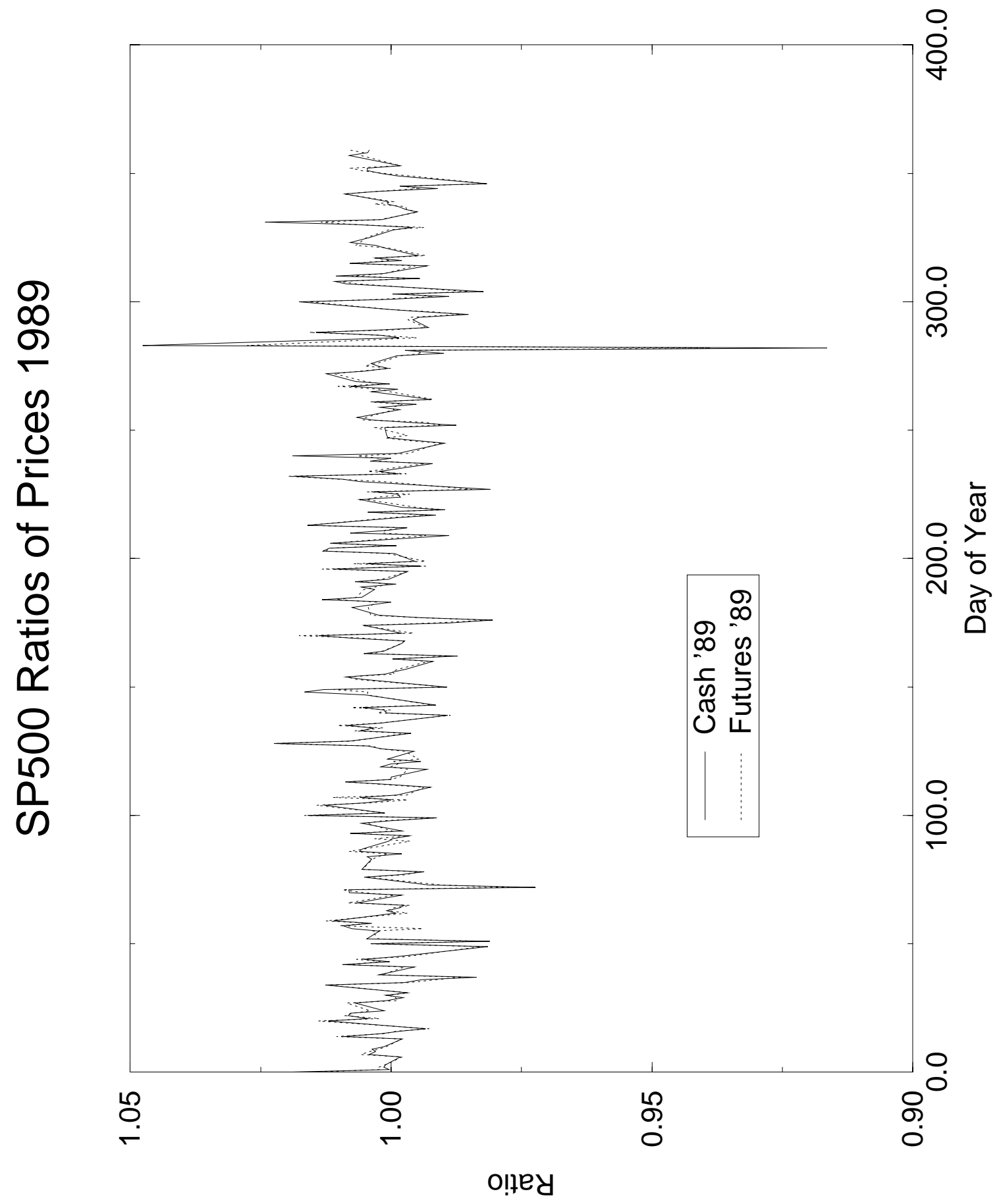




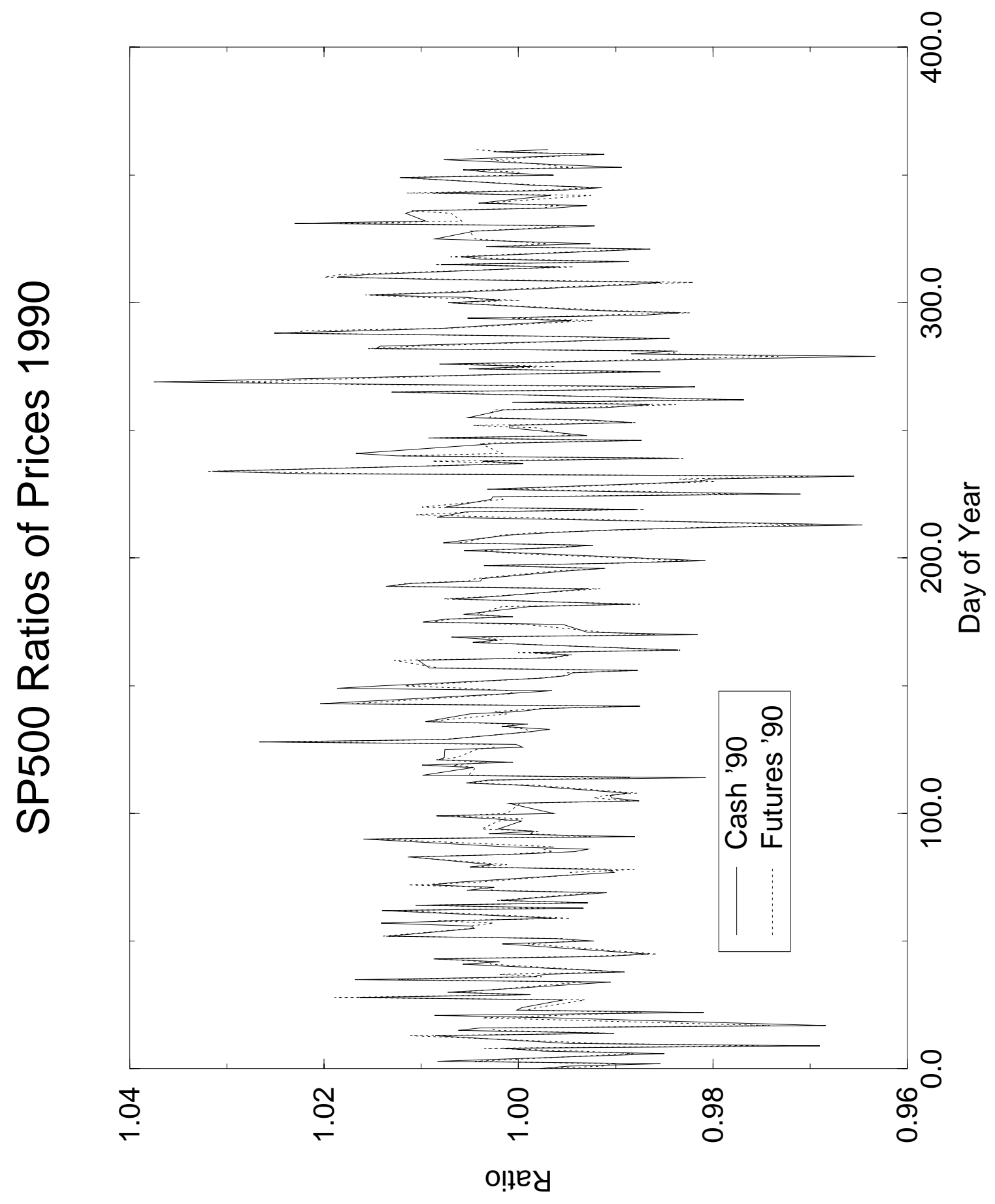




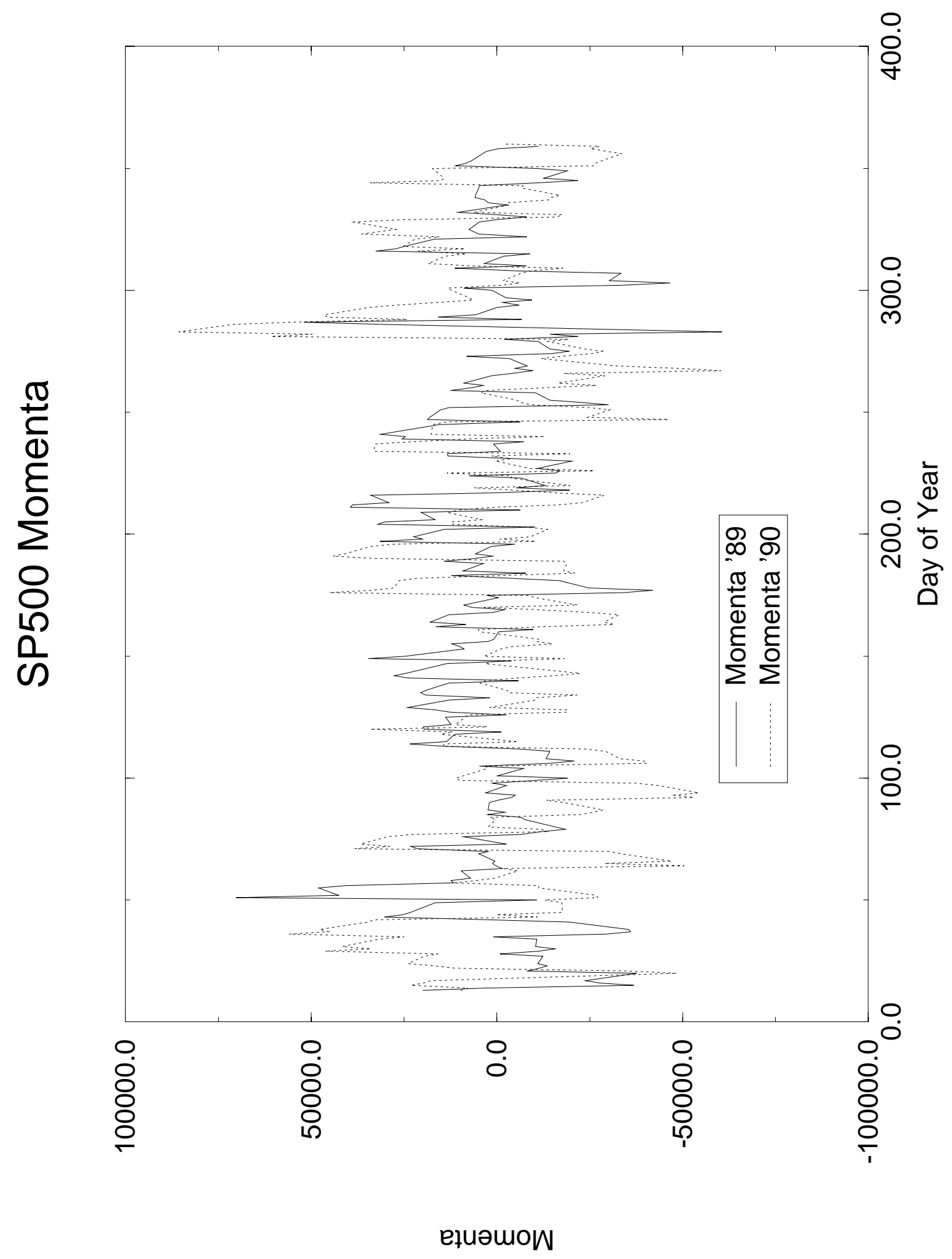




\begin{tabular}{|c|c|c|c|c|c|}
\hline Date & Action & Price & Momentum & Prof/Loss & Accum_P/L \\
\hline 900102 & 0 & 362.5 & 0 & 0 & 0 \\
\hline 900103 & 0 & 361.7 & 0 & 0 & 0 \\
\hline 900104 & 0 & 359.85 & 0 & 0 & 0 \\
\hline 900105 & 0 & 354.6 & 0 & 0 & 0 \\
\hline 900108 & 0 & 357.55 & 0 & 0 & 0 \\
\hline 900109 & 0 & 352.2 & 0 & 0 & 0 \\
\hline 900110 & 0 & 351.3 & 12888 & 0 & 0 \\
\hline 900111 & 0 & 351.85 & 16243 & 0 & 0 \\
\hline 900112 & 0 & 340.95 & 13635 & 0 & 0 \\
\hline 900115 & 0 & 339.2 & 9790.6 & 0 & 0 \\
\hline 900116 & 0 & 342.1 & 7712.6 & 0 & 0 \\
\hline 900117 & 0 & 338.75 & 23132 & 0 & 0 \\
\hline 900118 & 0 & 340.85 & 19694 & 0 & 0 \\
\hline 900119 & 0 & 342.2 & 17783 & 0 & 0 \\
\hline 900122 & -10 & 331.4 & -48359 & -0 & 0 \\
\hline 900123 & -10 & 330.25 & -35550 & 0 & 0 \\
\hline 900124 & 4 & 333.1 & 11288 & -920 & -920 \\
\hline 900125 & 4 & 326.75 & 16534 & 0 & -920 \\
\hline 900126 & 4 & 326.8 & 23973 & 0 & -920 \\
\hline 900129 & 4 & 326.7 & 19622 & 0 & -920 \\
\hline 900130 & 4 & 325.2 & 15954 & 0 & -920 \\
\hline 900131 & 4 & 330.5 & 46293 & 0 & -920 \\
\hline 900201 & 4 & 330.1 & 33920 & 0 & -920 \\
\hline 900202 & 4 & 332.5 & 41691 & 0 & -920 \\
\hline 900205 & 4 & 333.4 & 31801 & 0 & -920 \\
\hline 900206 & 4 & 330.25 & 25036 & 0 & -920 \\
\hline 900207 & 10 & 335.8 & 55905 & -0 & -920 \\
\hline 900208 & 10 & 335.3 & 45384 & 0 & -920 \\
\hline 900209 & 10 & 334.4 & 47744 & 0 & -920 \\
\hline 900212 & 10 & 330.75 & 34837 & 0 & -920 \\
\hline 900213 & 10 & 332.65 & 32927 & 0 & -920 \\
\hline 900214 & 4 & 333.3 & -10906 & -1320 & -2240 \\
\hline 900215 & 10 & 336.15 & 142.86 & -0 & -2240 \\
\hline 900216 & 4 & 333.2 & -17619 & -1545 & -3785 \\
\hline 900220 & 4 & 328.75 & -17480 & 0 & -3785 \\
\hline 900221 & 4 & 329.3 & -12886 & 0 & -3785 \\
\hline 900222 & 4 & 326.75 & -27091 & 0 & -3785 \\
\hline 900223 & 4 & 325.5 & -26829 & 0 & -3785 \\
\hline 900226 & 4 & 329.85 & -11259 & 0 & -3785 \\
\hline 900227 & 4 & 331.35 & -11244 & 0 & -3785 \\
\hline 900228 & 4 & 332.95 & 11798 & 0 & -3785 \\
\hline 900301 & 4 & 337.65 & 5833.8 & 0 & -3785 \\
\hline 900302 & 4 & 339.75 & 684.3 & 0 & -3785 \\
\hline 900305 & -10 & 338.45 & -5641.9 & -0 & -3785 \\
\hline 900306 & -10 & 343.2 & -1652.8 & 0 & -3785 \\
\hline 900307 & -10 & 340.9 & -50367 & 0 & -3785 \\
\hline 900308 & -10 & 344.5 & -29035 & 0 & -3785 \\
\hline 900309 & -10 & 342.05 & -46972 & 0 & -3785 \\
\hline 900312 & -10 & 342.65 & -33355 & 0 & -3785 \\
\hline 900313 & -10 & 339.55 & -30543 & 0 & -3785 \\
\hline 900314 & 4 & 341.35 & 38315 & -1520 & -5305 \\
\hline
\end{tabular}




\begin{tabular}{|c|c|c|c|c|c|}
\hline Date & Action & Price & Momentum & Prof/Loss & Accum_P/L \\
\hline 900315 & 4 & 342.2 & 28928 & 0 & -5305 \\
\hline 900316 & 4 & 345.25 & 36703 & 0 & -5305 \\
\hline 900319 & 4 & 347.6 & 29455 & 0 & -5305 \\
\hline 900320 & 4 & 345.6 & 22806 & 0 & -5305 \\
\hline 900321 & -10 & 342.2 & -14140 & -0 & -5305 \\
\hline 900322 & -10 & 339.1 & -11816 & 0 & -5305 \\
\hline 900323 & 4 & 340.8 & 2271.1 & 630 & -4675 \\
\hline 900326 & 4 & 341.75 & 449.86 & 0 & -4675 \\
\hline 900327 & 4 & 345.55 & 1952.8 & 0 & -4675 \\
\hline 900328 & 4 & 345.9 & -22453 & 0 & -4675 \\
\hline 900329 & 4 & 343.9 & -25895 & 0 & -4675 \\
\hline 900330 & 4 & 341.45 & -28679 & 0 & -4675 \\
\hline 900402 & 4 & 341.9 & -18134 & 0 & -4675 \\
\hline 900403 & 4 & 347.35 & -13281 & 0 & -4675 \\
\hline 900404 & -10 & 343.2 & -52988 & -0 & -4675 \\
\hline 900405 & -10 & 344.25 & -47766 & 0 & -4675 \\
\hline 900406 & -10 & 343.7 & -54351 & 0 & -4675 \\
\hline 900409 & -10 & 344.4 & -43528 & 0 & -4675 \\
\hline 900410 & -10 & 344.3 & -37378 & 0 & -4675 \\
\hline 900411 & 4 & 345.2 & 10026 & -1070 & -5745 \\
\hline 900412 & 4 & 348.1 & 11141 & 0 & -5745 \\
\hline 900416 & 4 & 346.85 & 2522.2 & 0 & -5745 \\
\hline 900417 & 4 & 347.2 & 3021.1 & 0 & -5745 \\
\hline 900418 & -10 & 342.9 & -40435 & -0 & -5745 \\
\hline 900419 & -10 & 339.6 & -39556 & 0 & -5745 \\
\hline 900420 & -10 & 336.4 & -33069 & 0 & -5745 \\
\hline 900423 & -10 & 332.65 & -29306 & 0 & -5745 \\
\hline 900424 & -10 & 332.45 & -23859 & 0 & -5745 \\
\hline 900425 & 4 & 334.25 & 14571 & 4255 & -1490 \\
\hline 900426 & 4 & 335.3 & 14083 & 0 & -1490 \\
\hline 900427 & 4 & 328.85 & -5697.1 & 0 & -1490 \\
\hline 900430 & 4 & 332.1 & 15090 & 0 & -1490 \\
\hline 900501 & 4 & 333.65 & 11924 & 0 & -1490 \\
\hline 900502 & 4 & 336.95 & 34151 & 0 & -1490 \\
\hline 900503 & 4 & 337.15 & 2677.8 & 0 & -1490 \\
\hline 900504 & 4 & 339.9 & 11210 & 0 & -1490 \\
\hline 900507 & 4 & 342.5 & 8524.8 & 0 & -1490 \\
\hline 900508 & 4 & 345.1 & 7836.9 & 0 & -1490 \\
\hline 900509 & 4 & 344.95 & -19098 & 0 & -1490 \\
\hline 900510 & 4 & 345.05 & -18684 & 0 & -1490 \\
\hline 900511 & 10 & 354.25 & 2082.6 & -0 & -1490 \\
\hline 900514 & 4 & 356.9 & -10305 & 1255 & -235 \\
\hline 900515 & 4 & 356.5 & -10375 & 0 & -235 \\
\hline 900516 & 4 & 355.35 & -21582 & 0 & -235 \\
\hline 900517 & 4 & 355.95 & -3070.7 & 0 & -235 \\
\hline 900518 & 4 & 355.6 & -2827.6 & 0 & -235 \\
\hline 900521 & 10 & 359 & 4474.5 & -0 & -235 \\
\hline 900522 & 4 & 360.8 & 3011.3 & 830 & 595 \\
\hline 900523 & 4 & 360.75 & -5706.7 & 0 & 595 \\
\hline 900524 & 4 & 359.85 & -15600 & 0 & 595 \\
\hline 900525 & 4 & 355.35 & -22373 & 0 & 595 \\
\hline
\end{tabular}




\begin{tabular}{|c|c|c|c|c|c|}
\hline Date & Action & Price & Momentum & Prof/Loss & Accum_P/L \\
\hline 900529 & 4 & 362.6 & 3146.1 & 0 & 595 \\
\hline 900530 & 4 & 363 & 140.75 & 0 & 595 \\
\hline 900531 & 4 & 361.75 & -18205 & 0 & 595 \\
\hline 900601 & 10 & 368.5 & 3596.1 & -0 & 595 \\
\hline 900604 & 4 & 373.75 & -1080.6 & 2555 & 3150 \\
\hline 900605 & 4 & 372.85 & -4107.9 & 0 & 3150 \\
\hline 900606 & 4 & 371 & -14972 & 0 & 3150 \\
\hline 900607 & 4 & 368.95 & -10720 & 0 & 3150 \\
\hline 900608 & 4 & 364.45 & -11414 & 0 & 3150 \\
\hline 900611 & 4 & 367.8 & 4533.4 & 0 & 3150 \\
\hline 900612 & 10 & 371.6 & 5086.7 & -0 & 3150 \\
\hline 900613 & -10 & 370.4 & -12051 & -600 & 2550 \\
\hline 900614 & -10 & 368.5 & -31489 & 0 & 2550 \\
\hline 900615 & -10 & 367.95 & -28874 & 0 & 2550 \\
\hline 900618 & -10 & 361.9 & -32799 & 0 & 2550 \\
\hline 900619 & -10 & 363.6 & -24803 & 0 & 2550 \\
\hline 900620 & -10 & 364.4 & -10826 & 0 & 2550 \\
\hline 900621 & 4 & 366.9 & 3518.8 & 1680 & 4230 \\
\hline 900622 & -10 & 360.15 & -21708 & -0 & 4230 \\
\hline 900625 & -10 & 357.6 & -10346 & 0 & 4230 \\
\hline 900626 & -10 & 355.95 & -8346.4 & 0 & 4230 \\
\hline 900627 & 4 & 359.45 & 44969 & 280 & 4510 \\
\hline 900628 & 4 & 362.15 & 33693 & 0 & 4510 \\
\hline 900629 & 4 & 362.35 & 27726 & 0 & 4510 \\
\hline 900702 & 4 & 364.4 & 26410 & 0 & 4510 \\
\hline 900703 & 4 & 364 & 21479 & 0 & 4510 \\
\hline 900705 & -10 & 359.8 & -21078 & -0 & 4510 \\
\hline 900706 & 4 & 362.25 & -17900 & -1295 & 3215 \\
\hline 900709 & -10 & 363.1 & -18866 & -0 & 3215 \\
\hline 900710 & -10 & 360.5 & -17949 & 0 & 3215 \\
\hline 900711 & 10 & 365.4 & 32718 & -1150 & 2065 \\
\hline 900712 & 10 & 369.45 & 44143 & 0 & 2065 \\
\hline 900713 & 10 & 370.9 & 40933 & 0 & 2065 \\
\hline 900716 & 10 & 372.3 & 34111 & 0 & 2065 \\
\hline 900717 & 10 & 370.4 & 27662 & 0 & 2065 \\
\hline 900718 & 4 & 367.15 & -10420 & 805 & 2870 \\
\hline 900719 & 4 & 368.45 & -797.44 & 0 & 2870 \\
\hline 900720 & 4 & 364.8 & -8972.7 & 0 & 2870 \\
\hline 900723 & -10 & 357.8 & -13582 & -0 & 2870 \\
\hline 900724 & 4 & 357.65 & -7943.9 & 5 & 2875 \\
\hline 900725 & 10 & 359.65 & 12270 & -0 & 2875 \\
\hline 900726 & 10 & 358.25 & 12179 & 0 & 2875 \\
\hline 900727 & 4 & 355.5 & 4115.4 & -2145 & 730 \\
\hline 900730 & 10 & 358.25 & 13536 & -0 & 730 \\
\hline 900731 & 10 & 358.6 & 10240 & 0 & 730 \\
\hline 900801 & 4 & 357.45 & 743.17 & -470 & 260 \\
\hline 900802 & -10 & 353.9 & -16462 & -0 & 260 \\
\hline 900803 & -10 & 346.65 & -23005 & 0 & 260 \\
\hline 900806 & -10 & 334.4 & -28930 & 0 & 260 \\
\hline 900807 & -10 & 337.2 & -16040 & 0 & 260 \\
\hline 900808 & 4 & 339.55 & -4669 & 7105 & 7365 \\
\hline
\end{tabular}




\begin{tabular}{|c|c|c|c|c|c|}
\hline Date & Action & Price & Momentum & Prof/Loss & Accum_P/L \\
\hline 900809 & 4 & 341.4 & 6135.7 & 0 & 7365 \\
\hline 900810 & 4 & 337.3 & -19696 & 0 & 7365 \\
\hline 900813 & 4 & 339.8 & -3751.1 & 0 & 7365 \\
\hline 900814 & 4 & 340.75 & -3971.4 & 0 & 7365 \\
\hline 900815 & 4 & 341.65 & 13427 & 0 & 7365 \\
\hline 900816 & -10 & 331.75 & -26107 & -0 & 7365 \\
\hline 900817 & -10 & 328.65 & -8952.2 & 0 & 7365 \\
\hline 900820 & 4 & 329.7 & 437.58 & 955 & 8320 \\
\hline 900821 & -10 & 323.5 & -3608.2 & -0 & 8320 \\
\hline 900822 & 4 & 317.2 & 1404.6 & 3080 & 11400 \\
\hline 900823 & 4 & 306.25 & -19699 & 0 & 11400 \\
\hline 900824 & 10 & 313 & 33077 & -0 & 11400 \\
\hline 900827 & 10 & 322.8 & 32852 & 0 & 11400 \\
\hline 900828 & 10 & 322.65 & 22551 & 0 & 11400 \\
\hline 900829 & 10 & 323.85 & 8138.7 & 0 & 11400 \\
\hline 900830 & 4 & 318.55 & -12864 & 2705 & 14105 \\
\hline 900831 & 10 & 322.55 & 17746 & -0 & 14105 \\
\hline 900904 & 10 & 327.95 & 17376 & 0 & 14105 \\
\hline 900905 & 10 & 328.55 & 13472 & 0 & 14105 \\
\hline 900906 & 4 & 324.4 & -46137 & 855 & 14960 \\
\hline 900907 & 4 & 327.4 & -24412 & 0 & 14960 \\
\hline 900910 & 4 & 325.1 & -30433 & 0 & 14960 \\
\hline 900911 & 4 & 325.4 & -24566 & 0 & 14960 \\
\hline 900912 & 4 & 325.7 & -9189.3 & 0 & 14960 \\
\hline 900913 & 4 & 321.9 & -6432.4 & 0 & 14960 \\
\hline 900914 & 4 & 319.45 & -5300.4 & 0 & 14960 \\
\hline 900917 & 4 & 321.1 & 4231.9 & 0 & 14960 \\
\hline 900918 & 4 & 321.6 & 3007 & 0 & 14960 \\
\hline 900919 & -10 & 318.7 & -13251 & -0 & 14960 \\
\hline 900920 & -10 & 314.4 & -26753 & 0 & 14960 \\
\hline 900921 & -10 & 314.6 & -16478 & 0 & 14960 \\
\hline 900924 & -10 & 307.3 & -29161 & 0 & 14960 \\
\hline 900925 & -10 & 311.3 & -17946 & 0 & 14960 \\
\hline 900926 & -10 & 308.3 & -60283 & 0 & 14960 \\
\hline 900927 & -10 & 302.7 & -48549 & 0 & 14960 \\
\hline 900928 & -10 & 306.75 & -32780 & 0 & 14960 \\
\hline 901001 & -10 & 318.25 & -11864 & 0 & 14960 \\
\hline 901002 & -10 & 318.35 & -17181 & 0 & 14960 \\
\hline 901003 & -10 & 313.7 & -25668 & 0 & 14960 \\
\hline 901004 & -10 & 315.3 & -28345 & 0 & 14960 \\
\hline 901005 & -10 & 314.85 & -23633 & 0 & 14960 \\
\hline 901008 & -10 & 317.4 & -13765 & 0 & 14960 \\
\hline 901009 & -10 & 305.75 & -19285 & 0 & 14960 \\
\hline 901010 & 4 & 302.2 & 60834 & 8180 & 23140 \\
\hline 901011 & 4 & 297.35 & 49809 & 0 & 23140 \\
\hline 901012 & 4 & 301.7 & 85671 & 0 & 23140 \\
\hline 901015 & 4 & 306 & 71221 & 0 & 23140 \\
\hline 901016 & 4 & 301.25 & 55437 & 0 & 23140 \\
\hline 901017 & 10 & 300.6 & 24137 & -0 & 23140 \\
\hline 901018 & 10 & 308.15 & 45642 & 0 & 23140 \\
\hline 901019 & 10 & 313.05 & 46494 & 0 & 23140 \\
\hline
\end{tabular}




\begin{tabular}{|c|c|c|c|c|c|}
\hline Date & Action & Price & Momentum & Prof/Loss & Accum_P/L \\
\hline 901022 & 10 & 315.5 & 34382 & 0 & 23140 \\
\hline 901023 & 10 & 313.8 & 27071 & 0 & 23140 \\
\hline 901024 & 10 & 315.45 & 15547 & 0 & 23140 \\
\hline 901025 & 10 & 311.4 & 6418.2 & 0 & 23140 \\
\hline 901026 & 10 & 306.25 & 7496.3 & 0 & 23140 \\
\hline 901029 & 10 & 304 & 12258 & 0 & 23140 \\
\hline 901030 & 10 & 306.2 & 13245 & 0 & 23140 \\
\hline 901031 & 4 & 306.8 & -1336.8 & 3030 & 26170 \\
\hline 901101 & 4 & 308.45 & -6118.9 & 0 & 26170 \\
\hline 901102 & 4 & 313.2 & -1520.1 & 0 & 26170 \\
\hline 901105 & 4 & 315.4 & -6488.9 & 0 & 26170 \\
\hline 901106 & 4 & 311.8 & -8829.9 & 0 & 26170 \\
\hline 901107 & -10 & 307.25 & -17689 & -0 & 26170 \\
\hline 901108 & 4 & 309.8 & 6536.9 & -1345 & 24825 \\
\hline 901109 & 10 & 315.55 & 18566 & -0 & 24825 \\
\hline 901112 & 10 & 320.5 & 13803 & 0 & 24825 \\
\hline 901113 & 4 & 319.15 & 8276.7 & 1730 & 26555 \\
\hline 901114 & 10 & 321.7 & 21227 & -0 & 26555 \\
\hline 901115 & 10 & 318.05 & 8577.3 & 0 & 26555 \\
\hline 901116 & 10 & 319.3 & 25329 & 0 & 26555 \\
\hline 901119 & 10 & 321.15 & 22045 & 0 & 26555 \\
\hline 901120 & 10 & 316.8 & 15453 & 0 & 26555 \\
\hline 901121 & 10 & 317.85 & 36481 & 0 & 26555 \\
\hline 901123 & 10 & 315.5 & 27101 & 0 & 26555 \\
\hline 901126 & 10 & 318.2 & 38527 & 0 & 26555 \\
\hline 901127 & 10 & 319.65 & 25526 & 0 & 26555 \\
\hline 901128 & 4 & 319.25 & -16117 & -1295 & 25260 \\
\hline 901129 & 4 & 316.75 & -17612 & 0 & 25260 \\
\hline 901130 & 4 & 324.05 & 6258.7 & 0 & 25260 \\
\hline 901203 & 4 & 327.15 & -3343.4 & 0 & 25260 \\
\hline 901204 & 4 & 330.95 & -2952.3 & 0 & 25260 \\
\hline 901205 & 4 & 334.5 & -13847 & 0 & 25260 \\
\hline 901206 & 4 & 333.25 & -14307 & 0 & 25260 \\
\hline 901207 & 4 & 330.9 & -16895 & 0 & 25260 \\
\hline 901210 & 4 & 332.25 & -6567.9 & 0 & 25260 \\
\hline 901211 & 4 & 331.15 & -7029.4 & 0 & 25260 \\
\hline 901212 & 10 & 334.1 & 34184 & -0 & 25260 \\
\hline 901213 & 10 & 332.5 & 15014 & 0 & 25260 \\
\hline 901214 & 10 & 329.65 & 14254 & 0 & 25260 \\
\hline 901217 & 10 & 329.15 & 16560 & 0 & 25260 \\
\hline 901218 & 10 & 333.1 & 17281 & 0 & 25260 \\
\hline 901219 & 4 & 331.9 & -26320 & -1170 & 24090 \\
\hline 901220 & 4 & 332.85 & -26496 & 0 & 24090 \\
\hline 901221 & 4 & 334.75 & -27842 & 0 & 24090 \\
\hline 901224 & 4 & 331.2 & -33629 & 0 & 24090 \\
\hline 901226 & 4 & 333.75 & -25192 & 0 & 24090 \\
\hline 901227 & 4 & 330.8 & -27657 & 0 & 24090 \\
\hline 901228 & 1 & 331.65 & -2317.3 & 0 & 24090 \\
\hline
\end{tabular}

